\title{
Unconstrained Face Recognition Quality: A Review
}

\author{
Ranbeer Tyagi ${ }^{1}$, Geetam Singh Tomar $^{2}$ and Laxmi Shrivastava ${ }^{3}$ \\ ${ }^{1}$ Research Scholar, Deptt. of Electronics \& Communication UTU, Dehradun, \\ India-248007 \\ ${ }^{2}$ THDC -IHET \\ Tehri Garhwal Uttarakhand, India-249124 \\ ${ }^{3}$ Deptt. Of Electronics \& Communication, MITS, Gwalior (M.P.) India-474005 \\ ranbeertyagi85@gmail.com, ${ }^{1}$ gstomar@ieee.org, ${ }^{3}$ lselex@yahoo.com
}

\begin{abstract}
This document signifies the overview of the Unconstrained Face Identification Quality within an unconstrained face-recognition atmosphere. It offers the functions like imbalance, lighting, and phrase variance strategy. It may be employed for both confirmation and id. At the moment, there are lots of means of front watch facerecognition. Couples of years ago, for computer-vision, numerous face-recognition methods were prepared. But, real-globe face-detection quiet deposit a challenging work. The interest in unconstrained useful face-recognition is growing using the detonation of online media for example neighborhood systems, and video-surveillance video wherever encounter evaluation is of great significance. Within this evaluation, we show up to manage reputation within the situation of graph presumption. We are able to identify a mystical encounter utilizing a Diverse Method. This research lights-out the options recommended for unconstrained face recognition quality region and recommending the clear answer to become used by RFG (Reference Face Graph) based face-recognition. Our purpose is RFG centered unconstrained face-recognition to improve the demonstration quality. The Formula and Simulator of recommended sensing methods is likely to be completed by utilizing Mat-laboratory.
\end{abstract}

Keywords:-Unconstrained face-recognition, Press selection, Accuracy, RFG, FRT, Network Security and Monitoring

\section{Introduction}

Nowadays has been the fantastic need for Picture Repair and face-recognition. The extremely study area has been face-recognition in computer-vision in support of the final mix of decades. Face-recognition methods are often employed for security areas apart from are progressively more getting used in numerous various uses. It may identify an absolute organization in an electronic picture by examining and evaluating designs that's a kind of biometric application purpose. For instance, utilizes facial-recognition to distinguish among people within the Kinetic movement gaming console. Face-recognition has been analyzed thoroughly; nevertheless, real world face-recognition nevertheless stays a difficult job. The interest in unconstrained useful face-recognition is increasing using the surge of online media for example internet sites, and video-surveillance-video wherever encounter evaluation is of substantial significance. We approach facerecognition within the framework of data theory. We identify an unfamiliar encounter utilizing an exterior Research Encounter Chart (REC). A RFG is produced and acknowledgement of the given encounter is attained by evaluating it towards the encounters within the built RFG.

In today day's the endemic globe, the requirement to corporeal belongings or even to proceed the security of info is complementary together progressively more substantial and 
progressively tougher. By second to second we pay attention to in regards to the offenses of acknowledgement license fraud, computer breaking's by hackers, or security breaches in an organization or management office stop [1]. In primarily of those offenses, within the traditional to utilize arrange methods the illegal's were fascinating advantage of a main problem: the businesses don't financing right of accessibility by "who we're", except by "what we've", such as for instance id permits, secrets, accounts, FLAG information's. No of those assets are now actually important people. Recently, ability turned available allowing verification of "correct" individual personality. The areas of "biometrics" are named this sort of engineering. Encased from the number of biometric recognition techniques, the physical methods (fingerprint, encounter, and genetics) are extra constant than methods in efficiency team (keystroke, voice-print). Face-recognition may be the distinctive of the few biometric techniques in order to get the characteristics of together raised precision and little intrusiveness. It's the precision of the physical show up to without any being unpleasant. As a result of this, unconstrained face-recognition has stretched the notice of students in places by security, mindset, and electronic imageprocessing, to processor eyesight. Several calculations have now been prepared meant for face-recognition [2]. While program protection and meal handle are it big extensively discussed uses, face-recognition has additionally proven useful in prior Disc in series meting out areas. The different programs of unconstrained face-recognition are of the encounters in various contexts for example monitoring confirming, neighbourhood marketing, and individual-pc conversation. It composed discover of routinely distinguishing or confirming an individual beginning a picture or movie rounds. Encounter verification or encounter approval may be the trouble of come to a decision whether acknowledged complement of pictures is of the comparable individual or not [3]. There are certainly a large amount of programs of face-recognition. We're referring to, below many use complete software such as for instance Encounter Id, Access-Control, Protection, Picture repository inspections, Common identity-verification, Monitoring, Simple people marking, Gambling, Cost evaluation, Producing psychological records, Distinguishing television shows, in-human computer conversation, Private Id - Passports, driver permits, Automatic id verification - edge handles, police force e.g., Movie surveillances, analysis, Individual Protection - driver checking program etc.,

\section{- $\quad$ Practical Uses and its Challenges}

Unconstrained face-recognition is of more practical use which is nevertheless challenging because of the next elements:

1. Present Variance: the facial skin to become acknowledged might approach beneath arbitrary poses [4]. The pretence and appear difference info may be used to evaluation the heftiness of encounter understanding calculations to phrase.

2. Imbalance: an automatic encounter sensor can be used for discovering the encounters [4], the gathered encounters aren't allied. Alternatively as primarily characteristic descriptors require agreement before characteristic elimination, imbalance humiliates the demonstration of the face-recognition framework. Easily available the amount of programs for picture agreement, for example following items on movie, motion research, along with a large amount of prior chores of computer-vision.

3. Lighting Variance: The illumination of the main topic of an artwork is definitely an insight component in producing an inventive component, and also the conversation of sunshine and darkness is just a valuable procedure within the performer's resource. Dispensation of lighting is just a substantial concept in computer artwork and computervision [4].

4. Phrase Variance: the facial skin pictures may vary with various words. In sign dispensation, entire dissimilarity denoising, as well thought to be entire difference 
regularization is just a process, commonly used in electronic image-processing, in order to have uses in clatter eradication.

5. Standard Blurs: take images of in coloring scenario are often fuzzy being an after effect of camera move, i.e., a motion of the camera while its hide is uncover. Most available deploring techniques reproduction the experiential fuzzy picture whilst the trouble of the sharp picture in the shape of a standard cloud primary.

This really is accustomed towards the precision of face-recognition strategies depreciates rapidly in unconstrained places [5]. This is often acknowledged to degradations developing by cloud, alter in lighting, present, and phrase, imperfect occlusions etc.,

We will find four Methods for face-recognition from blurry pictures could be categorized bellow.

(i) Deblurring- stay [6] whereby the research picture is originally deblurred and next second hand created for acknowledgement. About the other hand, deblurring artifacts really are a most significant reason for error especially for sensible to severe blurs.

(ii) Mixed deblurring and acknowledgement [7], the trunk which is computational trouble.

(iii) Obtaining cloud-invariant cosmetic look for acknowledgement [8]. Apart from these are effective simply for mild blurs.

(iv) The right acknowledgement progress of [9] whereby reblurred reviews by the gallery are compared from the blurry research picture.

It's substantial to notice using the purpose of each and every of the along with strategies assume a trusting room-invariant cloud reproduction.

For conduct illumination, present have mainly been two directions of detection-based sleeping on (i) the 9D subspace reproduction employed for encounter [10] and (ii) removing and corresponding lighting insensible cosmetic look [11-12]. Combine the ability of the around two techniques and recommend an incorporated framework to include an authentic illumination normalization stand for face-recognition below complex lighting scenario, Brown et. al., [13].

We take notice of the format and consistency limitations of the reproduction to become alienated by imaging restrictions, in support of face-recognition, for instance present and lighting. We explain both means of taking: Movie Imaging: It employs camcorders. Plan demonstration depends lying on correct placement, perspective of the head and regional lighting scenario. Thermal-Imaging: It along through cosmetic heat dissimilarity has additional precision \& it's excessively costly. 
- $\quad$ Fundamental setup of unconstrained Face-Recognition plan

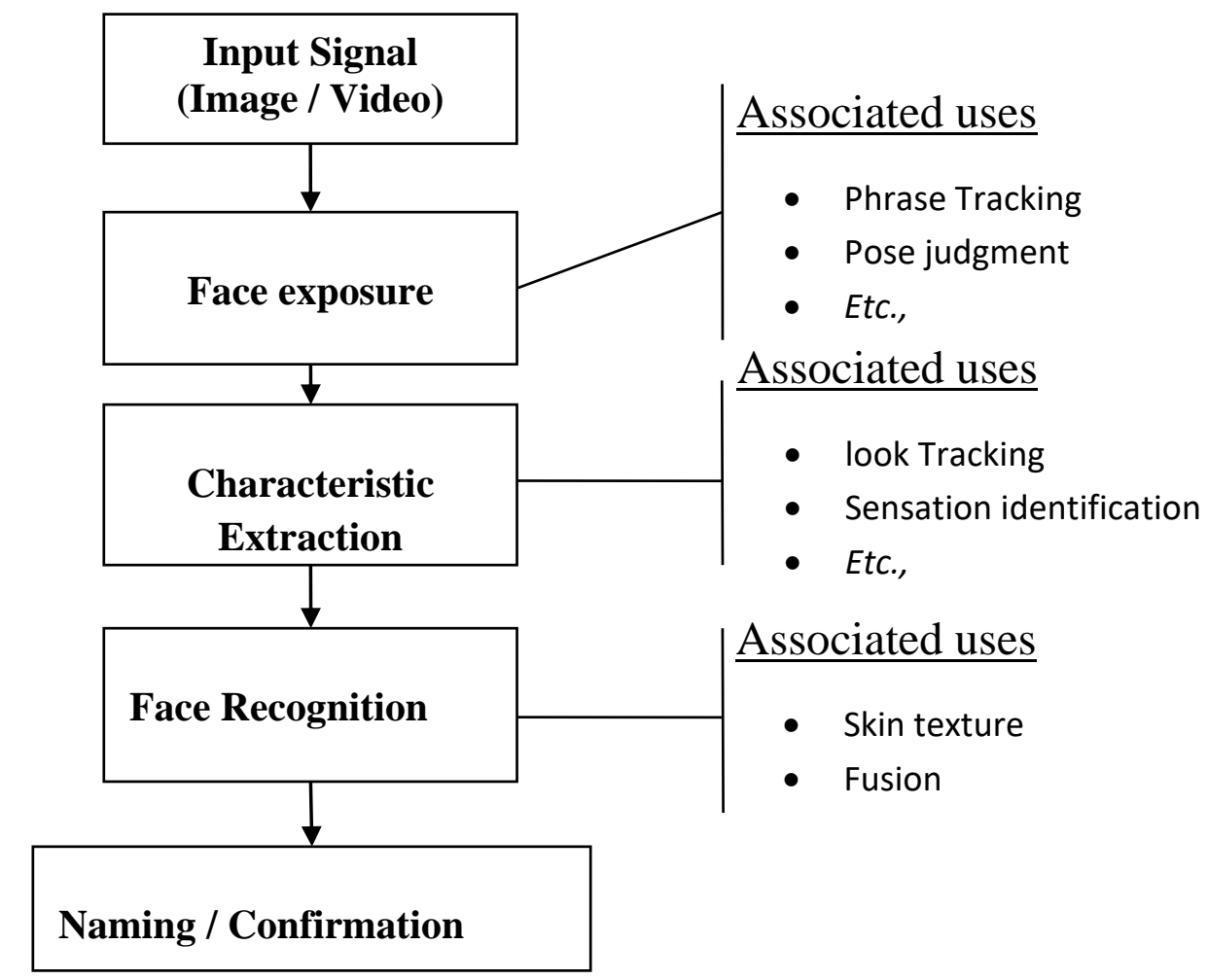

\section{Motivation}

Primarily existing facial-recognition strategies work by numeric key rules named encounter images. Such strategies identify 80 nodal factors on the person encounter. Within this scenario, nodal factors are end-points employed to decide adjustable of the person's encounter, as an example the duration or thickness of the nose, the profundity of the attention plugs and also the account of the nerve fillets. The facial skin printing may afterwards be used like a basis for distinction with info jailed by encounters within an impression or movie. The dissimilarity flanked by face-detection and acknowledgement is the fact that in recognition we instantly need to determine if easily available is just a quantity of encounters within the picture, apart from in acknowledgement we need to choose whose experience it's. We will find a lot of difficulties with the purpose of produce these challenging issues are picture degradations because of cloud and sound, and dissimilarities in search because of lighting and present. This really is also useful personally pc conversation, efficient fact, catalog rebirth, compact-disc, pc exercise, info security e.g., Face-recognition continues to be determined expansively; about the otherhand, real-planet face-recognition quiet locations a challenging objective. The state employed for unconstrained smart face-recognition is growing through the boom of online media for example public methods, and movie declaration saving anyplace encounter evaluation is of important significance. A RFG is created and verification of the recognized encounter is attained by contrasting it towards the encounters within the built RFG. Centrality procedures are using to identify personal encounters within the RFG. The motivation employed for having such areas is attached to the standard imbalance of encounter pictures after face-detection, in computation to using a size-free technique without the need for cosmetic attribute placement detection (e.g., eyes). Most express-of the-statue face-recognition calculations require combined encounters to trigger through raised precision. In the shape of manifold areas by different degree and transformation environments we expel need employed for encounter agreement. 


\section{Cosmetic Pictures with Versions}

Among the bulk large problems within the examiner area of picture acknowledgement is face-recognition [3]. An individual countenance isn't just a 3D item; it's as well a non rigid body. Furthermore, cosmetic pictures in many cases are obtained under environment. That's, the picture atmosphere might be excessively diverse and also the lighting scenario might be revolutionary. Figure 1, is definitely a situation of a picture having diverse environments.

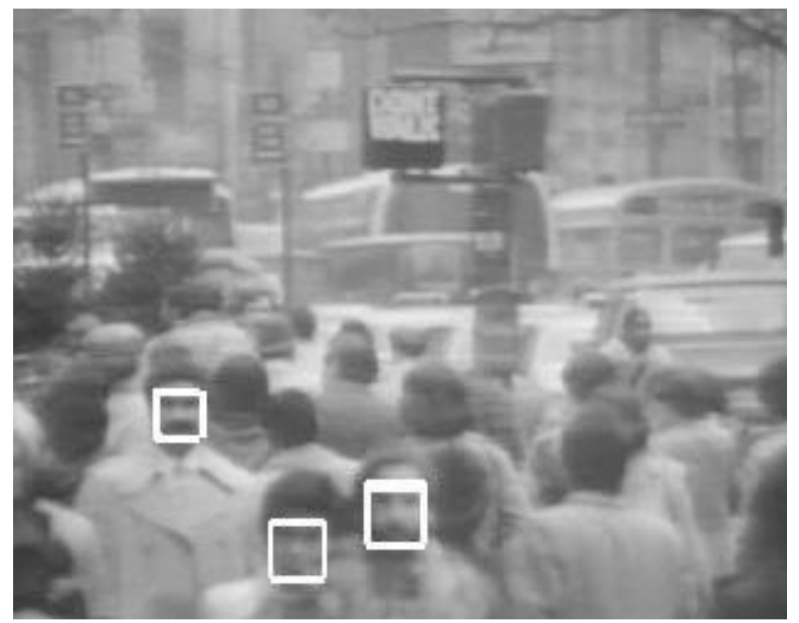

\section{Figure 1. The Facial Skin Sensor Discovered Three Encounters from the Diverse Environments}

The dissimilarities in cosmetic pictures might be classified the following:
a) Camera distortion and sound
b) Complicated history
c) Lighting
d) Interpretation, turn, climbing, and closure
e) Cosmetic phrase
f) structure and hairstyle

Camera distortion and sound are regular versions in picture identification issues. Prior scientists are suffering from numerous resources to improve the sign-to-noise rate. Within the path of agreement by diverse image environments, the recognizer requires a top quality encounter sensor to split up the particular encounters by additional fragments of the picture. Lighting is usually a main element in the impediment of the acknowledgement procedure. To ease the impact of the lighting impact, people might take traditional picture improvement methods (active thresholding, histogram equalization), or educate a sensory system meant for characteristic removal.

\section{Literarture Review}

\section{Common Review}

Often, Pictures could be private into two kinds: Restricted region images (Constrained domain images) and unconstrained region pictures (Unconstrained domain Images). These categorizations enable the picture to become calculated in a restricted environments 
anyplace the illumination and present is predetermined. Today existing isn't any difficulty of lighting and phony or perhaps a several extra trouble and also the picture is ready to identify. These types of pictures are recognized as the restricted pictures [55]. There are certainly a quantity of pictures someplace nothing is mounted. Present could be illumination and posturing evils. That's named the unconstrained site pictures. These pictures could be people being used with a distant camera or person's pictures of the pressing objective being used having a fixed camera. Every single one these images would be the unconstrained pictures. At the moment present could be acknowledgement evils. Present we need to reestablish the picture and then merely we are able to identify [55].

The miles flanked by substantial factors someplace aged to identify recognized encounters, e.g., calculating the area flanked from the eyes or extra substantial factors or calculating different perspectives of cosmetic system. Apart from its required towards the face-recognition strategies to become totally involuntary. We will find several facerecognition techniques. We're discussing below [14].

1. Alternative Corresponding Techniques: In alternative technique, absolutely the encounter area is being used interested in clarification as feedback info interested in encounter contagious plan. Individual of the best situation of alternative techniques are Eigenfaces [16], (most thoroughly utilized method created for face-recognition), Primary Component Evaluation (PCA), Linear Discriminant Analysis (LDA) [15] and impartial element evaluation (ICA) etc.,

2. Function-supported Strategies: within this approaches restricted characteristics for example eyes, nose and mouth are main of remove as well as their jobs in addition to restricted info are given interested in a structural classifier. A sizable face in support of function removal methods is attribute "reinstatement", this really is at what period the plan efforts to obtain back functions which are imperceptible because of large variations, ex. Head pretense at what period we're matching a forward picture with a drawing picture [17]. These procedures are discriminates among three kinds:

(i) Typical methods available on shapes (edges), sides, encounters and outlines.

(ii) Characteristic - pattern- based methods

(iii) Architectural harmonizing methods using the purpose of acquire interested in deliberation geometric limitations about the cosmetic look.

3. Blend Schemes: Blend face-recognition schemes start using a group of together alternative and show backed schemes. Often 3D Pictures are used in hybrid strategies. The picture of the people encounter is wedged in 3D, enabling the plan to notice the shapes of the attention hollows, such as for instance, normally the types of the cheek or eyebrow. Nevertheless a face in drawing might supply because the plan utilizes power, and a coalition of measurement, which supplies it adequate in series to construct an entire encounter. The 3D plan usually takings consequently: Acknowledgement, area Dimension, Diagnosis, Representation and Corresponding. Recognition - Taking an encounter additionally a checking take images of or shooting a people encounter in real instant.

\section{Literature Review of Associated Work}

Barkan et. al., In covering the 3D image will be distinction by an available 3D picture, it needs to possess no adjustments. Usually, about the other-hand, images using the purpose of are put in 2D, as well as in that covering, the 3D picture need a few adjustments. Present work centers around unconstrained face-recognition, that will be 
inherently complicated because of the unrestrained picture accomplishment to permits differences in present, lighting, phrase, and imbalance, form an over-entire encounter demo by personalized multiple-size LBP functions for experience recognition [18-19] Lei et. al., Research discriminant limited cosmetic look explained discriminant face descriptors (DFD) in an info - formidable style in its placement of the handcrafted technique which is effectiveness on together harmonized experience recognition and diverse face-recognition. Other to establishment in acknowledgement wherever largedimensional skin consistency aren't preferred, [20] Chen ETAL. The high-tech is achieved in experientially create apparent that in the shape of high-dimensional LBP attribute descriptors. To attempt the pretense versions, present-invariant face-recognition is achieved by with Markov random meadow stay picture harmonizing [21].

A probabilistic elastic harmonizing procedure is prepared to hold present dissimilarity in acknowledgement. Within plan, a Gaussian mixture model (design) (GMM) is employed to imprison the specific search sharing of the encounters within the planning placement and SVM is used created for encounter verification. To get rid of the lighting home using the reason for limit face-recognition buildings, an encounter example tell as Local Quantized Patterns (LQP). The illumination invariance of the demo instructions to improved demonstration created for high tech methods planned the challenging Labeled Faces in the Wild (LFW) report. In [22], the pictures of the comparable subject through different lingo are versioned being an assemblage of lay to sleep linked indicators and also the shortage economic documents for that dissimilarity in lexis. Writers recommend a newest restricted face-recognition plan to identify folks of interest by their fractional encounters. Finally, the similarity of two encounters is rehabilitated whilst the detachment among both of these allied characteristic jobs [23]. This determines the IARPA Janus Standard A (IJB-A), an overtly available method within the untamed dataset keeping 500 subjects through actually restricted to a little region encounter pictures. Detectives may subscribe to the delivering listing in support of info on such possibility discharges [24]. A photograph-precise legion normalization plan, available on polynomial degeneration, is prepared to improve the durability of encounter harmonizing below challenging circumstances. This record primarily provided a better taking of discriminative legion efficiency joining. In distinction, with "wild", unchecked cohort examples permit reaching the perfect demonstration [25].

The suggested a function of misinformation framework utilizing the group of SORT and alignment error (SORT-AE) to attain present invariant face-recognition [26]. This record available individual efficiency planned unconstrained nevertheless-to-still and movie-to-movie face harmonizing circumstances. They utilized the facial skin repository of two kinds, LFW and YTF [27]. COTS encounter exactly the same individually better the best face-recognition results earlier on the YTF report [28]. The restricted twin descriptors evaluated at the moment are restricted twin design, nearby stage quantization, and Binarized arithmetical picture skin consistency [29]. 2D and 3D Cosmetic recognition could be distinction laying about the source of different restrictions for example cosmetic expression versions, encounter path, lighting variations and trustworthiness. Apart from agreement $2 \mathrm{D}$ and $3 \mathrm{D}$ are utilized collectively next an improved demonstration is finished [30]. Solitary known view was using the reason for the HMAX-C2 attribute wasn't nearly as distress - difficult as have been elective by previous guides. We regional standard results for ten different face-recognition strategies about the vibrant dataset and challenge to its immediate to depart LFW and change onto extra difficult critiques for unconstrained face-recognition [31]. our data-set contains, an extensive assortment of difficulties checking occlusions, difficult poses, and small assertion and out-of-middle faces, the necessity of face areas as oblique areas and together grayscale and color pictures [32].A book face data put that'll create simple examine within the trouble of forward to stipulate face verification 'in the wild'. This information set could be named from the Superstars in Front-Profile (CFP) data-set. We analyzed the recital greater than several different 
calculations employing a restricted process and described how those all embarrass by Front-Front to Front-Account [33].

Writer provides the strategies for unconstrained face verification associated on cavernous convolutional skin consistency and determine it about the lately infinite IARPA Janus Benchmarks A (IJB-A) dataset along with about the traditional LFW dataset. We examine the efficiency of the DCNN method on lately unconfined challenging encounter verification info, IARPA Standard A, which retains faces through total present, illumination, and extra difficult circumstances [34]. The LFW report has been thoroughly used [35]. CRF-approximation segmentation is top quality adequate to provide an enormous agreement of in series regarding epidermis, hair, and environments as well as in computation may be used to approximation present [36]. We founded two book broad face-detection jobs, a filtered divorce of the initial PubFig details placement via a large amount of near -backup pictures separate. We anticipate that the great amount greater encounter collection may ultimately become available meant for examine factors [37]. The Distinction of Gaussian (PUPPY) jittering and Gabor wavelet psychoanalysis are distinction created for face-recognition by a picture development. RBF techniques are subjected to provide exceptional strength of recital [38]. The on line face id is preserved with a individual descriptor of the face picture being an inquiry. Author's sign up of way to their lately proven BHG descriptor, the SORT descriptor, and also the LBP descriptor [39].

The traditional understanding itself could be result in a typical experience recognizer. Recognition and restoration results are educational about the "Faces within the untamed" documents, which show the effectiveness of mutually traditional and online training [40].A Markov indiscriminate region is defined to create a smooth previous more than the restriction freedom and also the maximum a posteriori solution is calculated. It enables us to join up a newest customer via an individual 2D picture. Alternatively, we've regarded merely analysis pictures within the middle of personality that we've planning info [41]. The prepared plan is experienced on the neighborhood declaration video dataset with a three-camera program [42].

The item companions the one who scans from sideways a brief overview of the current publishing on face id in common and 3D encounter id in the shape of deepness feelers in careful [43]. Functionality is useful employing Multi-Layer consciousness. Checks are finished about the "Labeled Encounters within the Wild" info. Uncommon methods of creating haphazard dissimilarities of the planning info might as well manual to improvement [44]. An experience-picture, set-harmonizing transfer toward first of all urbanized and experienced about the "Labeled Encounters within the Wild" (LFW) standard that reproduces affects of face recognition by unconstrained pictures. The next efforts makes by recommended strategies. We show how marked problems examples, at what period available, may extra improve categorization demonstration, through the use of a just one of its-kind mixture - harmonizing funnel [45]. Writers recommend a newest plan for real-globe unconstrained present-invariant face-recognition. They principal develop a 3D reproduction for each subject within their report with simply an individual 2D picture by be related the 3D Universal Flexible Design strategy [46]. To the prepared formula behind moving the enter picture interested in twin form, the looking development shows the whole format of the facial skin. In the shape of the head placement and also the bulk typical length end-to-end of this last format we are able to determine the facial skin area through our plan [47]. This really is prone to produce sectioned graphic places. A gluttonous analysis formula appears for a demanding face candidate through centering its action in elliptical-much like places. A Length Change is put on section encounter functions [48].This manuscript provides a severe overview of analysis on picture-centered face-recognition crossways presents [49]. This analysis middle of interest on growing a face-recognition plan stay on Primary Component Evaluation and Self Organizing Maps unverified training formula [50]. 
We in work prevalent results on regular encounter requirements, along with a unique "in the wild" described dataset using the purpose of suggest our plan continue their stateof-the-art, from time to time somewhat [51]. In the prepared analysis work a technique on the basis of the group of Retinex and RECORD (DCT) plan is set up. For the reason that prepared analyze work the prepared plan is useful on extensive Yale $\mathrm{W}$ repository with simple LBP attribute elimination technique [52]. We've novice a book report, Marked faces in the Open, whose primary goals are; 1) offer an enormous record of real planet face pictures created for the concealed two of the type similar trouble of face id,

2) Wholesome so as interested in the recognition-positioning-acknowledgement funnel, and

3) Allow careful and easy distinction of face-recognition calculations [53].

Main, a book type of picture attribute, explained Normalized Pixel Distinction (NPD) is prepared. NPD attribute is determined whilst the dissimilarity to computation proportion caught between any two pixel power integrity, excited from the Weber part in investigational mindset [54]. An indistinct picture could be calculated like a problem reason for a sharp picture along with a form important component or PSF. Therefore in classify to recuperate the fast image we need to separate the image hooked on its smear important component and fast picture. Apart from this the problem at the moment may be the view of the cloud kernel [55].

In the given literature study it may be observed that to identify the facial skin phrase and enhancement of extra effective strategies for research collection variety. One more method would be to understanding attribute transformation or selection strategies to extra improve the demonstration of the prepared research encounter graph-based facerecognition. We prepared a narrative research face chart centered plan (such as for instance DCT, SVM and PCA etc.,) within the path of face-recognition in real world circumstances.

\section{Goals Review}

The goal of research as a result of multiplicity of face-recognition methods have now been prepared more than the historic times three years, some of these have accomplished encouraging efficiency, and they just function well under properly-controlled circumstances. In lots of real world reasons for example stylish declaration strategies in hectic places, individual faces are quite easily occluded by extra material such circumstances which is complex to obtain totally alternative encounter pictures for id. In the same period as normal, along with individual, features identification in assured circumstances might everlastingly be indefinable, existing motionless locations an enormous room among digital strategies and person demonstration on identifiable people. In other-hand safe for this room, large annotated situates of similes are preferred using the purpose of are envoy of the conclusion goals of unconstrained face-recognition.

- Incomplete Unconstraint Face Recognition Performance

1- To boost (Enhance) Quality guidelines in unconstrained face-recognition atmosphere for example precision (accuracy), Period effectiveness (efficiency), intellect, presence (visibility), PSNR and Edge Recognition.

2- To demonstration of the reference face graph-based unconstraint face-recognition regarding Quality Guidelines.

3- To handle unconstraint face-recognition underneath the combined home of non uniform cloud, lighting, Phrase variance and Present (pose) variations.

The next repository can be utilized for tests on picture in unconstrained face-recognition atmosphere such as for instance; LFW (Labeled faces in the Wild), Multiple-CAKE, FacePix, FEI and CMU-CAKE etc. 


\section{Conclusion}

We've provided an initial An Evaluation on Experience Recognition Methods. We've coated issues like the universal construction for face-recognition, facets that'll influence the efficiency of the recognizer, and many condition-of-the-artwork experience recognition methods. In real world circumstances, we prepared a unique RFG backed approach to face-recognition. Some encounter press may be used to higher a topic in forensic face-recognition. Some kind of present modification calculations must enhance the id precision. Anticipated outcome contains the Improvement of face-recognition methods with enhanced efficiency of the recommended, research encounter graph-based unconstrained face-recognition in image-processing with lighting and present variance. Our continuing work investigates complete algorithmic strategy of enhanced unconstrained face-recognition quality in unconstrained conditions.

Hopefully this document can offer the visitors a much better comprehension about-face reputation, and we motivate the visitors who're thinking about this subject to visit the referrals for more in depth research.

\section{References}

[1] Reuters News, "Computer Security Threat On Rise - U.S. Survey", March 7, 1999.

[2] R. Chellappa, C. L. Wilson and S. Sirohey, "Human and Machine Recognition of Faces: A Survey", Proc. IEEE, vol. 83, (1995) May, pp.705-741.

[3] T. Sim, S. Baker and M. Bsat, "The CMU pose, illumination, and expression (PIE) database," in IEEE International Conference on Automatic Face and Gesture Recognition, May (2002) 20-21, pp. 46-51.

[4] M. Kafai, L. An and B. Bhanu "Reference Face Graph for Face Recognition",IEEE Transactions on information forensics and security, vol. 9 , no. 12, (2014) September 19, pp $2132-2143$.

[5] A. Punnappurath, A. N. Rajagopalan, S.Taheri and R. Chellappa, "Face Recognition Across NonUniform Motion Blur, Illumination, and Pose" IEEE Transactions on image processing, vol. 24, no. 7, (2015) July 15.

[6] M. Nishiyama, A. Hadid, H. Takeshima, J. Shotton, T. Kozakaya and O. Yamaguchi, "Facial deblur inference using subspace analysis for recognition of blurred faces," IEEE Trans. Pattern Anal. Mach. Intell., vol. 33, no. 4, (2011) April, , pp. 838-845.

[7] H. Zhang, J. Yang, Y. Zhang, N. M. Nasrabadi, and T. S. Huang, "Close the loop: Joint blind image restoration and recognition with sparse representation prior," in Proc. Int. Conf. Comput. Vis, (2011) Novemeber, pp. $770-777$.

[8] R. Gopalan, S. Taheri, P. Turaga and R. Chellappa, "A blur-robust descriptor with applications to face recognition,” IEEE Trans. Pattern Anal. Mach. Intell., vol. 34, no. 6, (2012) June, pp. 1220-1226.

[9] P. Vageeswaran, K. Mitra and R. Chellappa, "Blur and illumination robust face recognition via settheoretic characterization,” IEEE Trans. Image Process., vol. 22, no. 4, (2013) April, pp. 1362-1372

[10] K.-C. Lee, J. Ho and D. Kriegman, "Acquiring linear subspaces for face recognition under variable lighting,” IEEE Trans. Pattern Anal. Mach. Intell., vol. 27, no. 5, (2005) May, pp. 684-698.

[11] S. Biswas, G. Aggarwal and R. Chellappa, "Robust estimation of albedo for illumination-invariant matching and shape recovery," IEEE Trans. Pattern Anal. Mach. Intell., vol. 31, no. 5, (2009) May, pp. 884-899.

[12] T. Zhang, Y. Y. Tang, B. Fang, Z. Shang and X. Liu, "Face recognition under varying illumination using gradientfaces," IEEE Trans. Image Process., vol. 18, no. 11, (2009) Novemeber, pp. 2599-2606

[13]X. Tan and B. Triggs, "Enhanced local texture feature sets for face recognition under difficult lighting conditions," in Analysis and Modeling of Faces and Gestures (Lecture Notes in Computer Science), Berlin, Germany: Springer-Verlag, , vol. 4778, (2007), pp. 168-182.

[14] Divyarajsinh N. Parmar, Brijesh B. Mehta, "Face Recognition Methods \& Applications" ,Int.J.Computer Technology \& Applications, vol 4, no. 1, (2013) January-Febuary, pp. 84-86

[15] S. Suhas, A. Kurhe and Dr.P. Khanale, "Face Recognition Using Principal Component Analysis and Linear Discriminant Analysis on Holistic Approach in Facial Images Database", IOSR Journal of Engineering e-ISSN: 2250-3021, p-ISSN: 2278-8719, vol. 2, no. 12, (2012) Decemebr. pp. 15-23.

[16]M. A. Turk and A. P. Pentland, "Face Recognition Using Eigenfaces", IEEE Computer Society Conference on Computer Vision and Pattern Recognition,. Proceedings CVPR '91, (1991) June, pp. 586 -591 ,

[17] S.-H. Lin, S.Y. Kung and L. J. Lin, "Face Recognition/Detection by Probabilistic Decision-Based Neural Network", IEEE Trans. Neural Networks, vol. 8, no. 1, (1997) January, pp.114-132.

[18] O. Barkan, J. Weill, L. Wolf and H. Aronowitz, "Fast high dimensional vector multiplication face recognition," in 13 Proceedings of the IEEE International Conference on Computer Vision (ICCV), (2013) Decemeber, pp. 1960-1967, 
[19]Z. Lei, M. Pietikainen and S. Li, "Learning discriminant face descriptor," IEEE Transactions on Pattern Analysis and Machine Intelligence, vol. 36, no. 2, (2014) Febuary, pp. 289-302,

[20] D. Chen, X. Cao, F. Wen and J. Sun, "Blessing of dimensionality: Highdimensional feature and its efficient compression for face verification," in Proceedings of the IEEE Conference on Computer Vision and Pattern Recognition (CVPR), (2013) June, pp. 3025-3032.

[21] S. Arashloo and J. Kittler, "Energy normalization for pose-invariant face recognition based on MRF model image matching," IEEE Transactions on Pattern Analysis and Machine Intelligence, vol. 33, no. 6, (2011) June. pp. 1274-1280,

[22] P. Li, Y. Fu, U. Mohammed, J. H. Elder and S. J. Prince, "Probabilistic models for inference about identity," IEEE Transactions on Pattern Analysis and Machine Intelligence, vol. 34, no. 1, (2012) January ,pp. 144-157,

[23]R. Weng, J. Lu and Y. P. Tan, "Robust Point Set Matching for Partial Face Recognition” IEEE transactions on image processing, vol. 25, no. 3, (2016) March.

[24] B. F. Klare, B. Klein, E. Taborsky, A. Blanton, J. Cheney, K. Allen, P. Grother, A.Mah, M. Burge and A. K. Jain, "Pushing the Frontiers of Unconstrained Face Detection and Recognition: IARPA" IEEE Conference on Computer Vision and Pattern Recognition (CVPR),pp. 7-12, (2015) June.

[25] M. Tistarelli, Senior Member, IEEE, Y. Sun and N. Poh, Member, IEEE, "On the Use of Discriminative Cohort Score Normalization for Unconstrained Face Recognition" IEEE Transactions on information forensics and security, vol. 9, no. 12, (2014) December.

[26] Y. Gao and H. J. Le, "Pose Unconstrained Face Recognition based on SIFT and Alignment Error" IEEE International Conference on Audio, Language and Image Processing (ICALIP), (2014), July, pp.277281.

[27] L. Best-Rowden, S. Bisht, J. C. Klontz and A. K. Jain, "Unconstrained Face Recognition: Establishing Baseline Human Performance via Crowd sourcing" IEEE International Joint Conference on Biometrics (IJCB), (2014) Septemeber 29-October 2-, pp. 1-8

[28] L. Best-Rowden, B.Klare, J. Klontz and A. K. Jain, "Video-to-Video Face Matching: Establishing a Baseline for Unconstrained Face Recognition",IEEE Sixth International Conference on Biometrics: Theory, Applications and Systems (BTAS), (2013) Septemebr 29- October-2, pp. 1-8,

[29] J.Ylioinas, A.Hadid, J. Kannala and M. Pietik"ainen, "An In-depth Examination of Local Binary Descriptors in Unconstrained Face Recognition" 22nd International Conference on Pattern Recognition (ICPR), (2014) August, pp. 4471-4476.

[30]R. Singh and H. Om, "An Overview of Face Recognition in an Unconstrained Environment" IEEE Second International Conference on Image Information Processing (ICIIP-2013), (2013) Decemeber, pp. 672-677.

[31] J. Z. Leibo, Q. Liao and T.Poggio, "Subtasks of Unconstrained Face Recognition" International Conference on Computer Vision Theory and Applications (VISAPP), vol.-2, pp. 113-121, Jan-2014.

[32] Vidit Jain and Erik Learned-Miller, "FDDB: A Benchmark for Face Detection in Unconstrained Settings" University of Massachusetts-Amhert-2010.

[33] S.Sengupta, J. C. Chen, C. Castillo, V. M. Patel, R. Chellappa and D. W. Jacobs, "Frontal to Profile Face Verification in the Wild" IEEE Winter Conference on Applications of Computer Vision (WACV), March (2016), pp. 1-9.

[34] J. C. Chen, V. M. Patel and R. Chellappa, "Unconstrained Face Verification using Deep CNN Features" IEEE Winter Conference on Applications of Computer Vision (WACV), (2016) March, pp.1-9.

[35] S. Liao, Z. Lei, D. Yi and S. Z. Li, "A Benchmark Study of Large-scale Unconstrained Face Recognition" IEEE International Joint Conference on Biometrics (IJCB), (2014) September 29- October 2, pp. $1-8$

[36] G. B. Huang, M. Narayana and E. L. Miller, “Towards Unconstrained Face Recognition” IEEE Computer Society Conference on Computer Vision and Pattern Recognition Workshops, (CVPRW '08), (2008) June, pp. 1-8.

[37] N. Pinto, Z. Stone, T. Zickler and D. Cox, "Scaling: A Case Study in Unconstrained Face Recognition on Facebook" CVPR-WORKSHOPS, (2011) June, , pp. 35-42.

[38]A. J. Howell and H. Buxton, "Towards Unconstrained Face Recognition from Image Sequences" Proceedings of the Second International Conference on Automatic Face and Gesture Recognition, (1996) October, pp. 224-229.

[39] J. W. H. Tangelder and B. A. M. Schouten, "Learning a Sparse Representation from Multiple Still Images for On-Line Face Recognition in an Unconstrained Environment" 18th International Conference on Pattern Recognition (ICPR'06), vol. 3, (2006), pp. 1086-1090.

[40]B. YAO, H. AI and S. LAO, "Person-Specific Face Recognition in Unconstrained Environments: a Combination of Offline and Online Learning" 8th IEEE International Conference on Automatic Face \& Gesture Recognition, (2008) September, pp.1-8.

[41] R. N. Rodrigues, G. N. Schroeder, J. J. Corso and V. Govindaraju, "Unconstrained Face Recognition using MRF Priors and Manifold Traversing" IEEE 3rd International Conference on Biometrics: Theory, Applications, and Systems, September (2009), pp. 1-9.

[42] L. An, M. Kafai and B. Bhanu, "Dynamic Bayesian Networkfor Unconstrained Face Recognitionin Surveillance CameraNetworks" IEEE Journal on emerging and selected topics in circuits and systems, 
vol. 3,no. 2, (2013) June, pp. 155-164.

[43] F. Schimbinschi, M. Wiering, R. E. MOHAN and J. K. SHEBA, "4D unconstrained real-time face recognition using a commodity depth camera" 7th IEEE Confrence on Industrial Electronics and Applications (ICIEA), (2012) July, pp. 166-173.

[44]A. Stormer and G. Rigoll, "learning weighted similarity measurements for unconstrained face recognition" 16th IEEE International Conference on Image Processing (ICIP), (2009) November, pp. 6164.

[45] L. Wolf, T. Hassner and Y. Taigman, "Effective Unconstrained Face Recognition by Combining Multiple Descriptors and Learned Background Statistics", IEEE Transactions on pattern analysis and machine intelligence, vol. 33, no. 10, (2011) October, , pp. 1978-1990.

[46] U. Prabhu, J. Heo and M. Savvides, "Unconstrained Pose-Invariant Face Recognition Using 3D Generic Elastic Models" IEEE transactions on pattern analysis and machine intelligence, vol. 33, no. 10 (2011) October, , pp. 1952-1961,

[47] S. M. Hafiz, Md. N. Hasan and Md. M. Islam, "An Efficient Scanning Based Learning Free Algorithm for Face Detection", International Conference on Infonnatics, Electronics \& Vision (ICIEV, May- 2012), pp. $52-56$.

[48] A. Cheddad, D. Mohamad and A. A. Manaf, "exploiting voronoi diagram properties in face segmentation and feature extraction", Journal Pattern Recognition, vol. 41, no. 12, Decemeber (2008), pp. 3842-3859.

[49] X. Zhang, and Y. Gao, "Face Recognition Across Pose: A review" Journal Pattern Recognition, vol. 42, no. 11, (2009) November, pp. 2876-2896.

[50]D. R. Anggraini, "Face Recognition Using Principal Component Analysis and Self Organizing Maps" Third ICT International Student Project Conference (ICT-ISPC-2014), (2014) March, pp. 91-94.

[51]H. Kaur and A. Kaur, "Illumination Invariant Face Recognition" International Journal of Computer Applications (0975 - 8887), vol. 64, no. 21, (2013) Febuary, pp. 23-27.

[52] G. B. Huang, M. Ramesh, T. Berg and E. L. Miller, "Labeled Faces in the Wild: A Database for Studying Face Recognition in Unconstrained Environments," Facial Image Processing and Analysis (FIPA), (2007) October, pp. 1-11.

[53] S. Liao, A. K. Jain and S. Z. Li, "A Fast and Accurate Unconstrained Face Detection," IEEE Transactions on Pattern Analysis and Machine Intelligence, vol. 38, no. 2, (2016) January, pp. 211-223.

[54] M. Poulose, "Literature Survey on Image Deblurring Techniques" International Journal of Computer Applications Technology and Research, vol. 2, no. 3, (2013), pp. 286 - 288.

[55] C. Indhumathi, "Unconstrained Face Recognition From Blurred and Illumination with Pose Variant Face Image Using SVM" International Journal of Innovative Research in Computer and Communication Engineering (An ISO 3297: 2007 Certified Organization), vol. 2, no. 1, (2014) March, pp. 2564-2567. 